\title{
FIBRA DE PIAÇAVA: ADSORVENTE ALTERNATIVO NA REMOÇÃO DE AZUL DE METILENO E PRETO REATIVO 5 DE SOLUÇÕES AQUOSAS
}

\author{
B.S.MARQUES ${ }^{1}$, G. L. DOTTO ${ }^{1}$, \\ ${ }^{1}$ Universidade Federal de Santa Maria, Departamento de Engenharia Química \\ E-mail para contato: bsmarques7@gmail.com
}

RESUMO - O presente trabalho teve por propósito avaliar a fibra de piaçava como adsorvente alternativo na remoção dos corantes azul de metileno (AM) e preto reativo5 (PR5) de soluções aquosas. Foram realizados estudos em relação a equilíbrio e termodinâmica, considerando ajuste dos modelos de Freundlich, Langmuir, Redlich-Peterson e Sips aos dados experimentais. Todos os ensaios foram conduzidos com volume, velocidade de agitação e quantidade de adsorvente iguais a $50 \mathrm{~mL}, 220 \mathrm{rpm}$ e $0,025 \mathrm{~g}$, respectivamente, durante 240 minutos. Para os estudos envolvendo equilíbrio, soluções aquosas dos corantes foram preparadas em concentrações de 30 a $300 \mathrm{mg} \mathrm{L}^{-1}$ e ensaios foram conduzidos nas temperaturas de 25,35 e $45^{\circ} \mathrm{C}$. Verificou-se que a capacidade de adsorção foi favorecida pelo aumento da temperatura, tanto em relação ao PR5, quanto ao AM, alcançando valores iguais a 223,9 $\mathrm{mg} \mathrm{g}^{-1}$ e 427,2 $\mathrm{mg} \mathrm{g}^{-1}$, respectivamente. $\mathrm{O}$ modelo de Redlich-Peterson mostrou-se mais adequado para caracterizar as isotermas de equilíbrio de ambos os corantes. Já os estudos termodinâmicos mostraram que a adsorção foi espontânea, favorável e endotérmica. Os resultados obtidos indicaram potencial na remoção de corantes através da adsorção com fibra de piaçava.

\section{INTRODUÇÃO}

Chave na economia brasileira, o setor têxtil constitui-se como uma significativa fonte de geração de empregos. Não obstante, os efluentes têxteis gerados representam um fator de risco tanto à população humana quanto ao meio ambiente, tendo em vista $o$ potencial mutagênico e carcinogênico de determinados compostos presentes nos efluentes produzidos. Dessa forma, órgãos regulamentadores vêm trabalhando no sentido de fomentar políticas que reduzam os impactos sociais e ambientais do setor têxtil, através de exigências legais que impliquem no tratamento adequado dos efluentes produzidos, previamente a seu descarte nos cursos d'água (Dotto et al., 2013).

Dentre as possibilidades disponíveis para remoção de corantes, a adsorção revelase uma opção interessante, devido ao baixo custo de operação aliado à eficiência na remoção de compostos indesejados. Dessa maneira, a busca por adsorventes abundantes e de baixo custo tornou-se contínua, a fim de reduzir ainda mais os custos do tratamento do efluente. Nesse sentido, uma alternativa seria a fibra de piaçava, resíduo proveniente de processos industriais voltados para a produção de vassouras, enchimento de assentos de carros, cordoaria e escovões (Avelar, 2008). 
Assim, este trabalho tem por intuito avaliar a fibra de piaçava quanto a seu potencial na remoção de azul de metileno e preto reativo 5, através da operação de adsorção, com enfoque nos estudos envolvendo equilíbrio e termodinâmica, bem como na determinação das características do adsorvente.

\section{MATERIAIS E MÉTODOS}

\subsection{Caracterizações do adsorvente}

O adsorvente foi adquirido com auxílio da Universidade Estadual de Santa Cruz/BA. A caracterização da fibra de piaçava deu-se através da microscopia eletrônica de varredura (MEV, (Jeol, JSM 6060, Japão)), e a identificação dos grupamentos funcionais ocorreu mediante análise de infravermelho com transformada de Fourier (FTIR, (Shimadzu, Prestige 21, Japão)).

\subsection{Ensaios de adsorção}

A adsorção foi realizada em incubadora (Shaker SL 222-SOLAB), a $220 \mathrm{rpm}$, sendo todos os ensaios conduzidos em soluções aquosas de volume igual a $50 \mathrm{~mL}$. Foram preparadas soluções de AM e PR5 nas concentrações de 30, 50, 100, 200 e 300 $\mathrm{mg} \mathrm{L}^{-1}$. Previamente à realização dos ensaios, foi efetuado o ajuste das soluções de AM e PR5 para os pHs 10 e 2, respectivamente. A quantidade de adsorvente em cada amostra foi fixada em $0,025 \mathrm{~g}$, de acordo com referências de estudos anteriores. Os ensaios ocorreram nas temperaturas de $25^{\circ} \mathrm{C}, 35^{\circ} \mathrm{C}$ e $45^{\circ} \mathrm{C}$, durante períodos de 240 minutos. As amostras coletadas nos ensaios foram filtradas e quantificadas em espectrofotômetro (UV mini-1240). As capacidades de adsorção ( $\mathrm{q}_{\mathrm{e}}, \mathrm{mg} \mathrm{g}^{-1}$ ) foram obtidas a partir da Equação 1:

$$
\mathrm{q}_{\mathrm{e}}=\frac{\left(\mathrm{C}_{0}-\mathrm{C}_{\mathrm{e}}\right)}{\mathrm{m}} \mathrm{V}
$$

onde, $\mathrm{C}_{0}$ e $\mathrm{C}_{\mathrm{e}}\left(\mathrm{mg} \mathrm{L}^{-1}\right)$ são as respectivas concentrações dos corantes no início e no equilíbrio, $\mathrm{m}$ (g) é a massa de adsorvente e V (L) é o volume de solução.

\subsection{Modelos de equilíbrio}

Para ajuste aos dados experimentais das isotermas, utilizaram-se os modelos de Freundlich (Freundlich, 1906), Langmuir (Langmuir, 1918), Sips (Sips, 1948) e RedlichPeterson, representados nas Equações 2, 3, 4 e 5, respectivamente:

$$
\mathrm{q}_{\mathrm{e}}=\mathrm{k}_{\mathrm{F}} \mathrm{C}_{\mathrm{e}}^{1 / n \mathrm{nF}} \text { (2) } \quad \mathrm{q}_{\mathrm{e}}=\frac{\mathrm{q}_{\mathrm{m}} \mathrm{k}_{\mathrm{L}} \mathrm{C}_{\mathrm{e}}}{1+\left(\mathrm{k}_{\mathrm{L}} \mathrm{C}_{\mathrm{e}}\right)} \text { (3) } \quad \mathrm{q}_{\mathrm{e}}=\frac{\mathrm{q}_{\mathrm{S}}\left(\mathrm{k}_{\mathrm{S}} \mathrm{C}_{\mathrm{e}}\right)^{\mathrm{m}}}{1+\left(\mathrm{k}_{\mathrm{S}} \mathrm{C}_{\mathrm{e}}\right)^{\mathrm{m}}} \text { (4) } \quad \mathrm{q}_{\mathrm{e}}=\frac{\mathrm{k}_{\mathrm{RP}} \mathrm{C}_{\mathrm{e}}}{1+\left(\mathrm{a}_{\mathrm{RP}} \mathrm{C}_{\mathrm{e}}\right)^{\beta}}
$$

onde $\mathrm{k}_{\mathrm{F}}\left(\left(\mathrm{mg} \mathrm{g}^{-1}\right)\left(\mathrm{mg} \mathrm{L}^{-1}\right)^{-1 / n F}, \mathrm{k}_{\mathrm{L}}\left(\mathrm{L} \mathrm{mg}^{-1}\right), \mathrm{k}_{\mathrm{S}}\left(\mathrm{L} \mathrm{mg}^{-1}\right)\right.$ e $\mathrm{k}_{\mathrm{RP}}\left(\mathrm{L} \mathrm{g}^{-1}\right)$ são as constantes de Freundlich, Langmuir, Sips e Redlich-Peterson, respectivamente. As capacidades máximas de adsorção dos modelos de Langmuir e Sips estão representadas por $\mathrm{q}_{\mathrm{m}}$ e $\mathrm{q}_{\mathrm{s}}$ $\left(\mathrm{mg} \mathrm{g}^{-1}\right), 1 / \mathrm{n}_{\mathrm{F}}$ corresponde ao fator de heterogeneidade, $\mathrm{m}$ é o expoente do modelo de Sips e $a_{R P}\left(\mathrm{~L} \mathrm{mg}^{-1}\right)^{\beta}$ e $\beta$ equivalem a constantes de Redlich-Peterson. 


\subsection{Parâmetros termodinâmicos}

Com o intuito de analisar o comportamento termodinâmico da adsorção, os valores das variações da energia livre de Gibbs $\left(\Delta \mathrm{G}^{0}, \mathrm{~kJ} \mathrm{~mol}^{-1}\right)$, entalpia $\left(\Delta \mathrm{H}^{0}, \mathrm{~kJ} \mathrm{~mol}^{-1}\right)$ e entropia $\left(\Delta \mathrm{S}^{0}, \mathrm{~kJ} \mathrm{~mol}^{-1} \mathrm{~K}^{-1}\right)$ foram estimados pelas Equações 6,7 e 8:

$$
\begin{aligned}
& \Delta \mathrm{G}^{\circ}=-\mathrm{RT} \ln \mathrm{K} \\
& \Delta \mathrm{G}^{\circ}=\Delta \mathrm{H}^{\circ}-\mathrm{T} \Delta \mathrm{S}^{\circ} \\
& \ln \mathrm{K}=\frac{\Delta \mathrm{S}^{\circ}}{\mathrm{R}}-\frac{\Delta \mathrm{H}^{\circ}}{\mathrm{RT}}
\end{aligned}
$$

onde $\mathrm{T}$ é a temperatura $(\mathrm{K})$; $\mathrm{R}$ é a constante universal dos gases $\left(8,314 \mathrm{~J} \mathrm{~mol}^{-1} \mathrm{~K}^{-1}\right)$ e $\mathrm{K}$ é a constante de equilíbrio.

\section{RESULTADOS E DISCUSSÃO}

\subsection{Características do adsorvente}

As Figuras 1 e 2 apresentam respectivamente as imagens de MEV e o espectro FTIR das fibras de piaçava. As Figuras 1 (a) e 1 (b) mostram que a piaçava tem uma estrutura fibrosa, composta por fibrilas menores. Os comprimentos das fibras são variados e o diâmetro é na ordem de 80-100 micrômetros. No espectro FTIR da Figura 2, as principais bandas foram observadas em 3300, 2950, 1750 e na região de 1500$1000 \mathrm{~cm}^{-1}$. A banda intensa em $3300 \mathrm{~cm}^{-1}$ pode ser atribuída ao estiramento de ligações $\mathrm{OH}$ presentes na lignina, celulose ou hemicelulose. A banda em $2950 \mathrm{~cm}^{-1}$ é relativa a vibrações simétricas ou assimétricas de $-\mathrm{CH}_{2}$. A banda em $1750 \mathrm{~cm}^{-1}$ está relacionada com o $\mathrm{C}=\mathrm{O}$ da carbonila, que juntamente com o ombro ao redor de $1100 \mathrm{~cm}^{-1}$ sugere a presença de ácidos, ésteres ou lactonas ligadas a anéis aromáticos. A banda na região de $1000 \mathrm{~cm}^{-1}$ é relativa aos estiramentos de $\mathrm{C}-\mathrm{O}$ da lignina, celulose ou hemicelulose ou ainda C-O-C da celulose ou hemicelulose. Na região de $1500-1000 \mathrm{~cm}^{-1}$ podem-se observar as vibrações de anéis guaiacílicos da lignina. Estes grupos funcionais podem ser potenciais sítios para a adsorção de corantes, fazendo da fibra de piaçava uma alternativa viável.

Figura (1) - Imagens de MEV das fibras de piaçava.

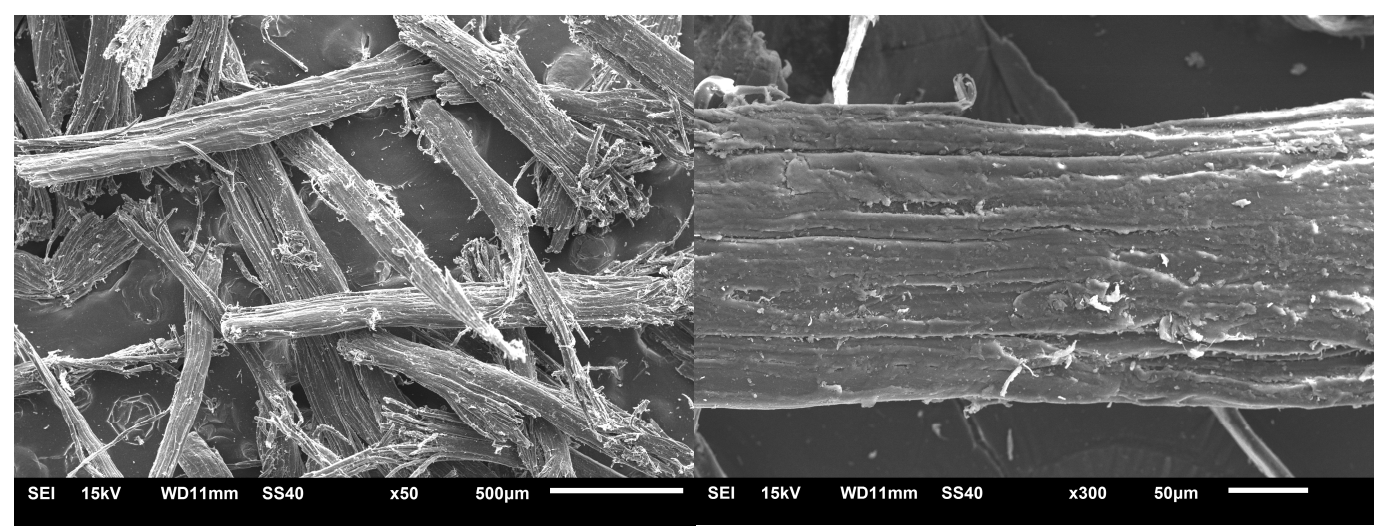

(a)

(b) 
Figura (2) - Espectro FTIR das fibras de piaçava.

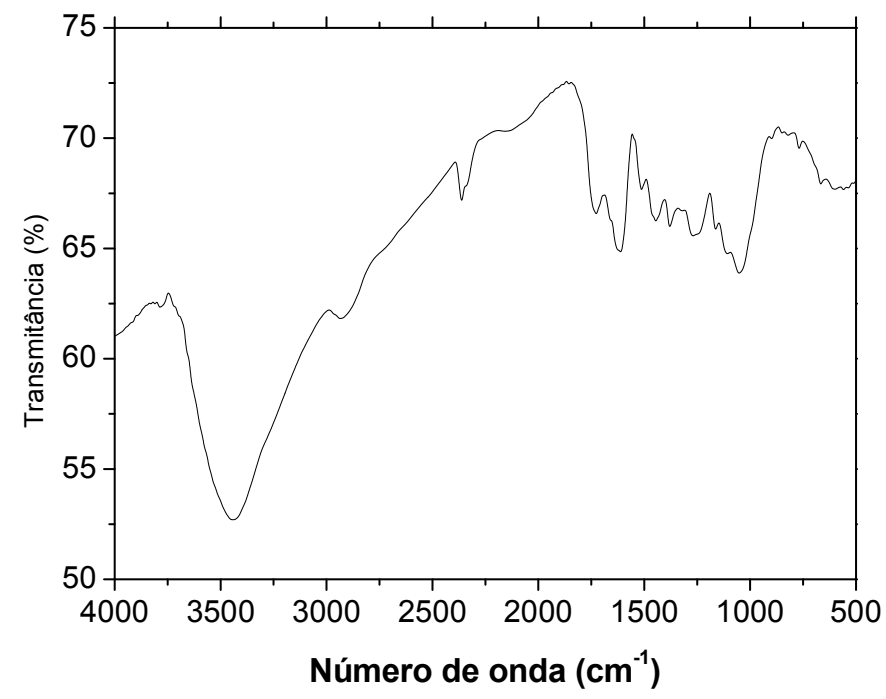

\subsection{Isotermas de equilíbrio}

Na Figura (3), são apresentadas as isotermas de adsorção para o azul de metileno (a) e preto reativo $5(\mathrm{~b})$, nas temperaturas de $25^{\circ} \mathrm{C}, 35^{\circ} \mathrm{C}$ e $45^{\circ} \mathrm{C}$. Observa-se na Figura (3) que, em ambos os casos, a capacidade de adsorção é favorecida pelo aumento da temperatura. Assim, as capacidades máximas de adsorção foram observadas em $45^{\circ} \mathrm{C}$, atingindo 223,9 $\mathrm{mg} \mathrm{g}^{-1}$ para o PR5 e 427,9 $\mathrm{mg} \mathrm{g}^{-1}$ para o AM. Especialmente no caso do AM, nota-se que em comparação à capacidade máxima de adsorção obtida para outros materiais a $25^{\circ} \mathrm{C}$, como palha de milho $\left(102,8 \mathrm{mg} \mathrm{g}^{-1}\right)$ e resíduo do palmito pupunha $\left(50,9 \mathrm{mg} \mathrm{g}^{-1}\right)$ (Honorato et al., 2015) e fibra de ouricuri $\left(29,8 \mathrm{mg} \mathrm{g}^{-1}\right)$ (Silva et al, 2015), $\mathrm{o}$ desempenho alcançado com a fibra de piaçava revelou-se muito superior $\left(328,8 \mathrm{mg} \mathrm{g}^{-1}\right)$.

Figura (3) - Isotermas de equilíbrio e modelo Redlich-Peterson ajustado aos pontos experimentais, em 25,35 e $45^{\circ} \mathrm{C}$, para a) AM e b) PR5.

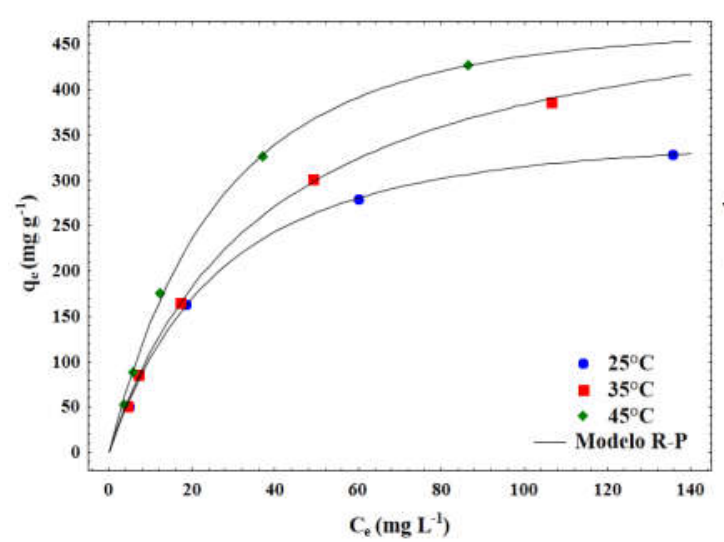

(a)

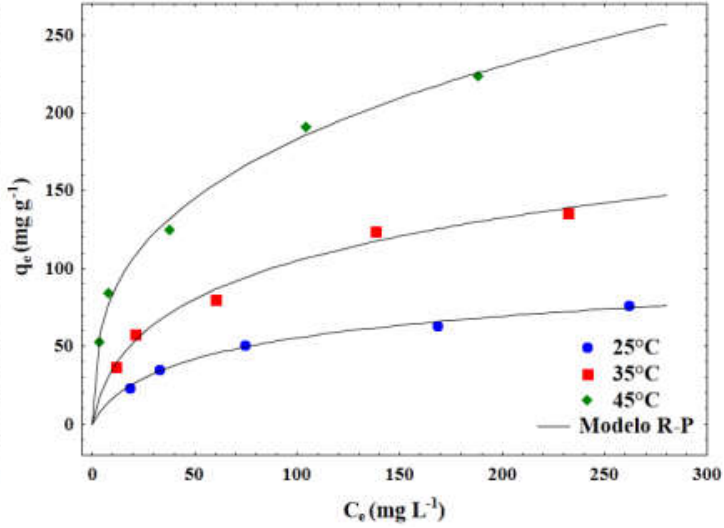

(b) 
Ainda na Figura (3), a capacidade de adsorção observada no $\mathrm{AM}$ a $45^{\circ} \mathrm{C}$ foi superior a do PR5 na mesma temperatura. É provável que, sendo o PR5 maior em sua estrutura molecular do que o AM, a interação com o adsorvente tenha sido dificultada, reduzindo assim a capacidade de adsorção. Observação similar foi realizada em trabalho de Dotto et al(2013), durante estudo sobre a adsorção de corantes alimentícios em filmes de quitosana.

Os parâmetros dos modelos ajustados aos dados experimentais constam na Tabela 1 , bem como os respectivos valores de $\mathrm{R}^{2}$ e EMR. A partir da Tabela 1, faz-se perceptível que tanto o modelo de Redlich-Peterson quanto o de Sips poderiam descrever adequadamente os pontos experimentais de ambos os corantes, tendo em vista os valores do coeficiente de determinação $\left(\mathrm{R}^{2}>0,99\right)$ e erro médio relativo $($ EMR $<4,7$ $\%)$.

Tabela 1- Parâmetros de equilíbrio para a adsorção do AM e do PR5.

\begin{tabular}{|c|c|c|c|c|c|c|}
\hline \multirow[t]{2}{*}{ Temperatura } & \multicolumn{3}{|c|}{ Azul de metileno } & \multicolumn{3}{|c|}{ Preto reativo 5} \\
\hline & $25^{\circ} \mathrm{C}$ & $35^{\circ} \mathrm{C}$ & $45^{\circ} \mathrm{C}$ & $25^{\circ} \mathrm{C}$ & $35^{\circ} \mathrm{C}$ & $45^{\circ} \mathrm{C}$ \\
\hline Freundlich & & & & & & \\
\hline $\mathrm{K}_{\mathrm{F}}\left(\mathrm{mg} \mathrm{g}^{-1}\right)\left(\mathrm{mg} \mathrm{L}^{-1}\right)$ & 40,8 & 35,5 & 42,7 & 8,67 & 16,03 & 37,5 \\
\hline $1 / \mathrm{n}_{\mathrm{F}}$ & 0,44 & 0,52 & 0,53 & 0,392 & 0,400 & 0,343 \\
\hline $\mathrm{R}^{2}$ & 0,964 & 0,981 & 0,974 & 0,991 & 0,988 & 0,996 \\
\hline $\operatorname{EMR}(\%)$ & 17,61 & 14,18 & 17,93 & 4,95 & 6,19 & 4,44 \\
\hline Langmuir & & & & & & \\
\hline $\mathrm{q}_{\mathrm{m}}\left(\mathrm{mg} \mathrm{g}^{-1}\right)$ & 399,6 & 521,6 & 579,5 & 87,6 & 159,8 & 228,8 \\
\hline $\mathrm{k}_{\mathrm{L}}\left(\mathrm{L} \mathrm{mg}^{-1}\right)$ & 0,04 & 0,03 & 0,03 & 0,019 & 0,023 & 0,054 \\
\hline $\mathrm{R}^{2}$ & 0,998 & 0,999 & 0,998 & 0,994 & 0,984 & 0,957 \\
\hline EMR (\%) & 4,36 & 2,88 & 5,35 & 2,70 & 6,70 & 13,29 \\
\hline Redlich-Peterson & & & & & & \\
\hline $\mathrm{k}_{\mathrm{RP}}\left(\mathrm{L} \mathrm{g}^{-1}\right)$ & 13,1 & 13,9 & 17,4 & 2,49 & 7,09 & 161,51 \\
\hline$a_{R P}\left(L_{m g}^{-1}\right)^{\beta}$ & 0,019 & 0,026 & 0,016 & 0,079 & 0,179 & 3,894 \\
\hline B & 1,00 & 0,99 & 1,00 & 0,823 & 0,753 & 0,675 \\
\hline $\mathrm{R}^{2}$ & 0,999 & 0,999 & 0,999 & 0,997 & 0,991 & 0,996 \\
\hline EMR (\%) & 3,40 & 2,93 & 3,44 & 2,73 & 4,64 & 4,01 \\
\hline Sips & & & & & & \\
\hline $\mathrm{q}_{\mathrm{mS}}\left(\mathrm{mg} \mathrm{g}^{-1}\right)$ & 370,9 & 502,8 & 518,4 & 105,9 & 220,8 & 774,5 \\
\hline $\mathrm{k}_{\mathrm{S}}\left(\mathrm{L} \mathrm{mg}^{-1}\right)$ & 0,04 & 0,03 & 0,04 & 0,012 & 0,009 & 0,001 \\
\hline $\mathrm{m}_{\mathrm{S}}$ & 1,14 & 1,04 & 1,15 & 0,772 & 0,686 & 0,418 \\
\hline $\mathrm{R}^{2}$ & 0,999 & 0,999 & 0,999 & 0,996 & 0,992 & 0,996 \\
\hline EMR (\%) & 2,73 & 2,44 & 1,70 & 2,93 & 4,61 & 3,86 \\
\hline
\end{tabular}

\subsection{Análise termodinâmica}

A Tabela 2 apresenta os parâmetros termodinâmicos para a adsorção de AM e PR5, estimados a partir do modelo de Redlich-Peterson.

Verifica-se na Tabela 2 que, para os dois corantes, a adsorção foi espontânea e favorável, a julgar pelos valores negativos de $\Delta G^{0}$. É também assinalável que os valores de $\Delta G^{0}$ tendem a ficar ainda mais negativos conforme o aumento da temperatura, sendo a adsorção mais favorecida em $45^{\circ} \mathrm{C}$. Os valores positivos de $\Delta \mathrm{H}^{0}$ e $\Delta \mathrm{S}^{0}$ indicam que a adsorção é endotérmica e que a desordem aumenta após a operação. 
Tabela 2 - Parâmetros termodinâmicos.

\begin{tabular}{cccc}
\hline Temperatura $\left({ }^{\circ} \mathrm{C}\right)$ & $\begin{array}{c}\Delta \mathrm{G}^{0} \\
\left(\mathrm{~kJ} \mathrm{~mol}^{-1}\right)\end{array}$ & $\begin{array}{c}\Delta \mathrm{H}^{0} \\
\left(\mathrm{~kJ} \mathrm{~mol}^{-1}\right)\end{array}$ & $\begin{array}{c}\Delta \mathrm{S}^{0} \\
\left(\mathrm{~kJ} \mathrm{~mol}^{-1} \mathrm{~K}^{-1}\right)\end{array}$ \\
\hline \multicolumn{4}{c}{ Azul de metileno } \\
\hline 35 & $-23,5$ & 0,12 \\
45 & $-24,3$ & 11,2 \\
\hline \multicolumn{4}{c}{ Preto reativo 5 } \\
\hline 25 & $-25,8$ & 0,61 \\
\hline 5 & $-19,3$ & 163,1 \\
\hline
\end{tabular}

\section{CONSIDERAÇÕES FINAIS}

Para ambos os corantes, verificou-se que a adsorção foi favorecida pelo aumento da temperatura de operação, com capacidades máximas de adsorção registradas em $45^{\circ} \mathrm{C}$, sendo iguais a $427,9 \mathrm{mg} \mathrm{g}^{-1}$ e $223,9 \mathrm{mg} \mathrm{g}^{-1}$ para AM e PR5, respectivamente. Nos dois casos, o modelo de Redlich-Peterson revelou-se como mais conveniente para ajuste das isotermas de adsorção. A adsorção foi espontânea, favorável e endotérmica. Em vista de todos estes aspectos, pode-se afirmar que a fibra de piaçava apresenta potencial para retenção de corantes têxteis em meio aquoso.

\section{REFERÊNCIAS BIBLIOGRÁFICAS}

AVELAR, F.F.; Utilização de fibra de piaçava na preparação de carvões ativados. 2008. 88f. Dissertação (Mestrado em Agroquímica). Universidade Federal de Lavras, Minas Gerais. 2008.

DOTTO, G.L.; MOURA, J.M; CADAVAL, T.R.S. ; PINTO, L.A.A. Application of chitosan films for the removal of food dyes from aqueous solutions by adsorption. Chemical Engineering Journal, v.214, p.8-16, 2013.

FREUNDLICH, H.; Over the adsorption in solution, Z. Phys. Chem. A57, p. 358471, (1906).

HONORATO, A. C., MACHADO, J. M., CELANTE, G., BORGES, W. G., DRAGUNSKI, D. C.; CAETANO, J. Biossorção de azul de metileno utilizando resíduos agroindustriais. Rev. Bras. Eng. Agríc.Ambiental, v. 19, n. 7, p. 705-710, 2015.

LANGMUIR, I.; The adsorption of gases on plane surfaces of glass, mica and platinum;J. Am. Chem. Soc. 40; p. 1361-1403; (1918).

REDLICH, O.; PETERSON, D. L.; A useful adsorption isotherm, J. Chem. Phys. 63; p. 1024-1027.1959.

SILVA, T. S. D; HENRIQUE, D. C; MEILI, L.; SOLETTI, J. I.; CARVALHO, S. H.A. D. Utilização da fibra de ouricuri (Syagrus coronata) na remoção do corante azul de metileno: variáveis de processo e isoterma de adsorção. Congresso Brasileiro de Sistemas Particulados. São Carlos, 2015.

SIPS, R. J. Chem. Phys., v. 16, p. 490- 495, 1948. 